\title{
ECOLOGY AND DIVERSITY OF WILDLIFE IN THE ECO-PARK OF THE JAMUNA BRIDGE AND ITS ADJACENT AREA, SIRAJGONJ, BANGLADESH
}

\author{
Hasibur Rahman, M. Firoj Jaman ${ }^{1 *}$ and Md. Saidur Rahman \\ Department of Zoology, Jagannath University, Dhaka-1100, Bangladesh \\ ${ }^{1}$ Department of Zoology, University of Dhaka-1205, Bangladesh \\ *Email:mfjaman@yahoo.com
}

\begin{abstract}
Animal diversity of an area is a good indicator for understanding a healthy habitat. We conducted a detail study on ecology and the wildlife diversity in the Eco-park of Jamuna bridge and its adjacent area of Sirajgonj district from April 2010 to March 2011. We employed transect line method and interviewed local people to reveal the status and distribution of wild animal and identify their microhabitats. A total of 89 species of wild animals was recorded, of which, 6 (6.74\%) species were amphibians, $11(12.36 \%)$ reptiles, $56(62.93 \%)$ birds and $16(17.94 \%)$ mammals. Regarding relative abundance, 18 (20.22\%) species of wildlife were very common, 35 (39.33\%) common, $28(31.46 \%)$ fairly common, $7(7.87 \%)$ few and only one $(1.12 \%)$ was occasionally found. In total 25 species of wildlife were identified as threatened category. In amphibians, 3 species were vulnerable nationally. In reptiles, 4 species were vulnerable and one endangered. In birds, 4 species were vulnerable, 6 endangered and one critically endangered (Gallicrex cinerea). In mammals, 3 vulnerable and 3 endangered nationally. Among the amphibians, skipper frog (Euplyctis cyanophlyctis) and toad (Bufo melanostictus) frequently occurred. Among the reptiles, common garden lizard (Calotis versicolor), common skink (Mabuya carinata) and cheekered keel back water snake (Xenochorphis piscator) were frequently occurred. Among the birds, common myna (Acrodotheres tristis), pied myna (A. fuscus), black drongo (Dicrurus macrocercus) and house crow (Corvus splendens) occurred frequently. Among the mammals, shrew (Suncus murinus), flying fox (Pteropus giganteus) and black rat (Rattus rattus) frequently occurred. Our study shows that illegal exploitation of trees, overgrazing of domestic animals, shooting and collection of young animals are the mentionable causes for declining wild animals. We suggest that awareness creation and preparation of proper management action plan in cooperation with related national and international organizations are necessary in order to protect the wildlife resources in the study area.
\end{abstract}

Key words: Wildlife diversity, habitat.

\section{INTRODUCTION}

Diversity of wildlife may increase by the conservation and protection of habitat and declaration of eco-park or protected areas with the help of the government and non-government organizations. However, environmental degradation has been occurring in a faster rate due to the rapid increase of human activities, 
interference to the wilderness areas, overexploitation, deforestation and natural calamities like soil erosion, tornados, etc. The study area contains a variety of unique habitats of wild animals. Every day many visitors come to the place for recreation and enjoy themselves observing the natural beauty. In this regard, wildlife diversity may increase the natural beauty as well as help to keep natural balance as they are important biotic component of the ecosystem. Many species of wild animals act as an important biological pest control agent e.g., insectivore and rodentivore.

Little work was done on the ecology, population status and habitats of some wildlife at Sirajgonj and adjacent areas by Sarker and Sarkar (1985) and Husain and Sarker (1971, 1979). Although, some works on the similar topic were done elsewhere (Haque 1975, Akter 1977, Banerjee 1978, Penafiel 1995, Rosario and Hai 1996, Jasmin 1996, Hossain and Sarker 1997, Sarker et al. 2001, Jaman et al. 2004). Detail collection of data on wildlife in the Eco-park at Jamuna Bridge and near the study site was not done in the past. It is necessary to have the baseline data of all information of wildlife of a habitat in order to make any management plan for their conservation. For this reason, authors were interested to conduct a detail study on wildlife diversity, status and their ecology in the Eco-park at Jamuna Bridge.

\section{STUDY AREA AND VEGETATION}

Sirajgonj district belongs to Rajshahi division with an area of about $2497.92 \mathrm{~km}^{2}$. The main rivers are Jamuna, Baral, Ichamati, Karatoya and Phuljuri. The district is bounded by Bogra district on the north, Pabna district on the south, Tangail and Jamalpur districts on the east, Pabna, Natore and Bogra districts on the west. The Eco-Park is a part of the North Bangel and located in the Jamuna Bridge of Saydabad Upazila in the Sirajgonj Sadar district. The park is controlled by Pabna Forest
Range. It covers currently an area of 600 ha (coordinates). The park was established through a gazette notification in 1998-1999.

The park is transected by a highway, a railway, a bridge and a power transmission corridor, the rest house; the forest beat office and the other institute in the middle of the park. In the Eco-Park trees were planted which are now thriving and growing into a forest. The annual average temperature reaches a maximum of $34.6^{\circ} \mathrm{C}$ and a minimum of $11.90^{\circ} \mathrm{C}$. The annual rainfall is $1610 \mathrm{~mm}$. The dominant plants are: Shisu (Dalbergia sissoo), jam (Eugenia jambolana), mehoginy (Switenia mahogoni), babla (Acacia arabica), debdaru (Polyalthia longifolia), bandarlathy (Cassia fistula), kadam (Anthocephalus chinensis), woodapple (Aegle marmelos), coconut (Cocos nucifera), tetul (Tamarindus indica), betelnut (Areca catechu), amloki (Emblica officinalis), tulsi (Ocimum sanctum), margosa tree (Azadirachata indica), muktajuhri (Acalypha indica), arohor (Cajanus cajan), horitoki (Terminalia chebula), krishnachura (Delonix regia), nalkhagra (Phragmites karka).

\section{MATERIALS AND METHODS}

The study was conducted from April, 2010 to March, 2011 at Jamuna Eco-park and its adjacent area. Different species of wild fauna was recorded during the study period. Data collection was done once in a month (6-7 days) and continued from early morning to evening during the whole study period. We employed transact line method, plotting method, direct observation for data collection and information collected through the interview with local people.

Transact line: In this method wildlife were observed and counted on each side of the transact line (size: $1000 \mathrm{~m}$ long $\times 100 \mathrm{~m}$ width) using tape recorder. A total of 60 transect lines were made across the study site. 
Plot counting: A total of sixty plots (size: $500 \mathrm{~m} \times$ $100 \mathrm{~m}$ ) were selected to study the wildlife, particularly amphibians and mammals within the study site. A total of 75 days were spent in the field. Observation was started early in the morning and ended at late afternoon in each day. During observation, movement of observer was kept at a uniform speed and while walking along a route, attempts were made to note the animals when they were whiting, singing or flying over the study area or foraging and feeding either on the ground or on the trees. Climber, bamboo ladder and portable hide were used for closer observation. During observation and data collection field notes, two pairs of binoculars (Prism a $10 \times 50$ ), digital camera (Cannon auto focus $35 \mathrm{~mm}$ and $16 \mathrm{~mm}$ ), National Video Camera, GPS, distance measuring plastic tape, surgical gloves, preservative and chemicals, plastic and metal tapes, map, paper box and rubber tapes, etc. were used. We also used field guide on birds (Ali and Ripley 1968-74, 1983), amphibians and reptiles (Daniel 1983, Smith 1931, 1943) and mammals (Prater 1993) in order to identify species during observation and also in the laboratory for identifying collected specimens and pictures. Museum species of wild animals of the Department of Zoology, University of Dhaka was also used to identify collected specimens and pictures of wild animals taken from the field. Data collected in each trip imputed in the computer to make the systematic data base for each species and categorized according to the taxonomic position. Habitat types, food habits, etc were also recorded.

\section{RESULTS AND DISCUSSION}

In the present study a total of 89 species of wild animals were recorded. Of these, 6 species were amphibians, 11 reptiles, 56 birds and 16 mammals (Table 1).

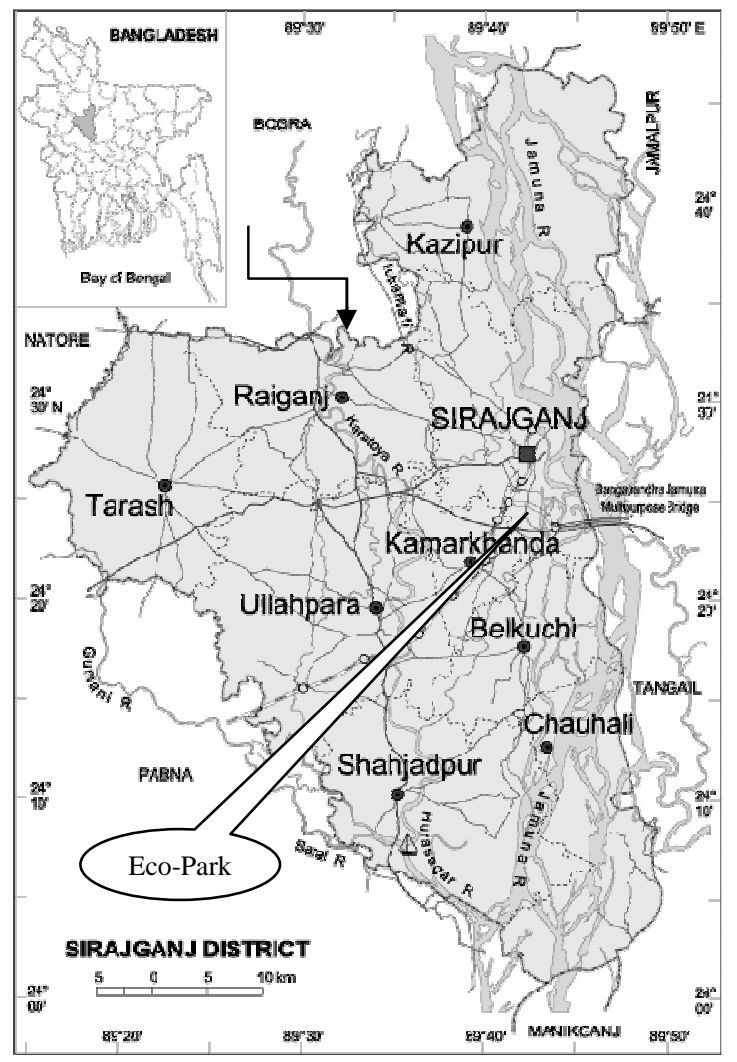

Fig. 1. Map of Sirajgonj district indicating the study site.

\section{Amphibians}

A total of 6 species of amphibians under 3 families and one order were observed in the Ecopark and its adjacent area. Among the wildlife, amphibians represented $6.74 \%$ species (Fig. 2). Relative abundance showed that two species were very common, 1 common and 3 were few in number (Fig. 3). The population of Bufo melanostictus showed the highest density (17.33 indiv./ha) and Rana cyanophlyctis showed the lowest density (4.0 indiv./ha) (Table 1). According to IUCN (2000) category, 3 species of amphibians were vulnerable at national level. Of the amphibians, three species occurred on aquatic and terrestrial habitats, 1 species on aquatic and semiaquatic habitats like river, pond, ditches, wetland, water edge, etc. and 2 species on the terrestrial habitats like, bush, open place, human habitation, 
cultivated land, tall tree, hole, etc. for foraging, nesting and roosting activities (Table 1, Figs. 4 and $5)$.

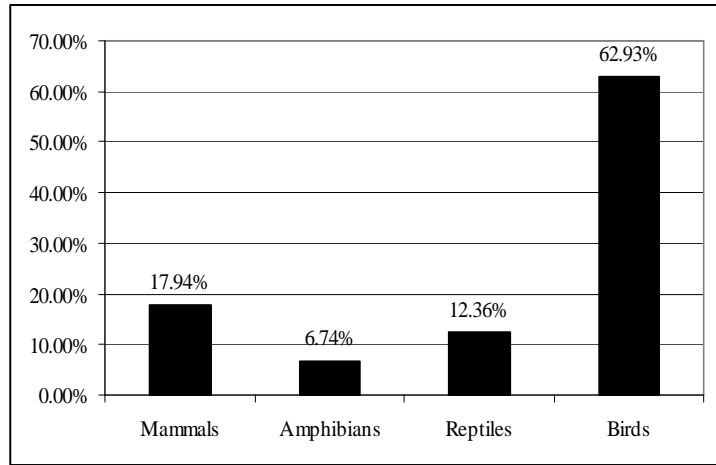

Fig. 2. Species diversity of wildlife in the Eco park.

\section{Reptiles}

A total of 11 species of reptiles were recorded. Of the reptilian species, 9 were lizards and 2 species of snakes (Table 1). Of the wild fauna, reptiles constituted $12.36 \%$ species (Fig. 1). Among the reptiles, 7 were common, 1 fairly common, 1 few and 2 very common (Fig. 3). The population of Hemidactylus brookii showed the highest density (20.5 indiv/ha) and Varanus flavescens showed the lowest density (1.0 indiv./ha) (Table 1). In the present study, 5 $(45.45 \%)$ species of reptiles were included in the threatened category, of which 1 (9.09\%) endangered and $4(36.36 \%)$ vulnerable at national level. Sarker and Sarker (1983) and IUCN (2000) reported that 22 species of reptiles are threatened in Bangladesh. Only one species of reptile used aquatic and terrestrial habitats, 1 species aquatic and semi-aquatic habitats like river, pond, ditches, wetland, water edge, etc. and 9 species used terrestrial habitats like bush open place, human habitation, cultivated land, tall tree, hole, etc. were used as feeding, nesting and roosting sites (Table 1, Figs. 4 and 5).

\section{Birds}

Fifty six species of birds belonging to 12 orders and 22 families were observed in the EcoPark and adjacent areas, of which 55 species were resident and only one winter migrant (Table 1). Among these, 36 species were non passerines and 20 passerines. Of the wild animals, birds constituted $62.93 \%$ species (Fig. 2). Regarding relative abundance of species, 14 species were very common, 24 common, 16 fairly common and 2 species were few (Fig. 3). The population of Passer domesticus showed the highest density (125.17 indiv./ha) and Sterna aurantia showed the lowest density (1.83 indiv./ha) (Table 1). Thirty species of birds are threatened nationally (Sarker and Sarker 1988). In the present study, 11 (19.64\%) species of birds are included in the threatened categories, of which $1(1.79 \%)$ critically endangered, $6(10.71 \%)$ endangered and 4 (7.14\%) vulnerable at national level (Table 1). A total of 2 species of birds used aquatic and terrestrial habitats, 13 used aquatic and semi-aquatic habitats like river, pond, ditches, wetland, water edge, etc. and 41 species used terrestrial habitats like bush, open place, human habitation, cultivated land, tall tree, hole, etc. for feeding, nesting and roosting activities (Table 1, Figs. 4 and 5).

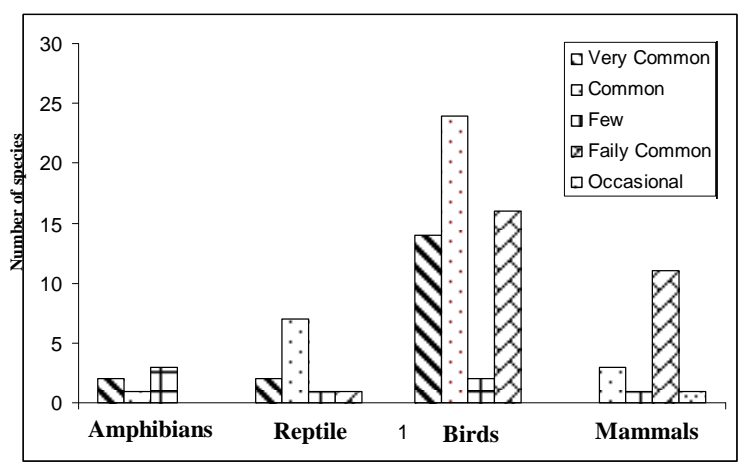

Fig. 3. Relative abundance of wildlife species in the Eco park. 


\section{Mammals}

A total of 16 species of mammals belonging to 9 families and 6 orders were recorded in the EcoPark and adjacent area. Mammals constituted $17.94 \%$ among the total wildlife (Fig. 2). Regarding relative abundance, 11 species were fairly common, 3 common, only one few and one occasional (Fig. 3). The population of Rattus rattus showed the highest density (19 indiv./ha) and Lepus nigricollis showed the lowest density (0.83 indiv./ha) (Table 1). Sarker and Sarker (1986) and IUCN (2000) reported that fifteen species of mammals are threatened nationally. In the present study, $6(37.50 \%)$ species of mammals are included in the threatened categories, of which 2 endangered and 4 species are vulnerable at national level. A total of two species of mammals used aquatic and terrestrial habitats and 14 species used terrestrial habitats like bush, open place, human habitation, cultivated land, tall tree, hole, etc. for display of feeding, nesting and roosting activities (Table 1, Figs. 4 and 5).

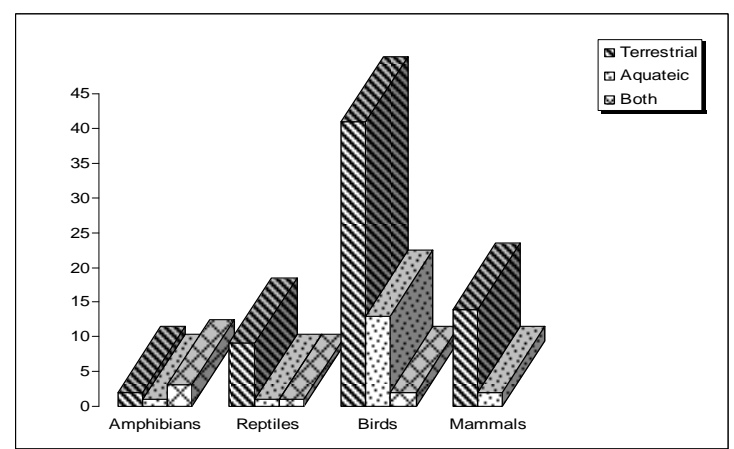

Fig. 4. Macro-habitat utilization by wildlife species in Eco park.

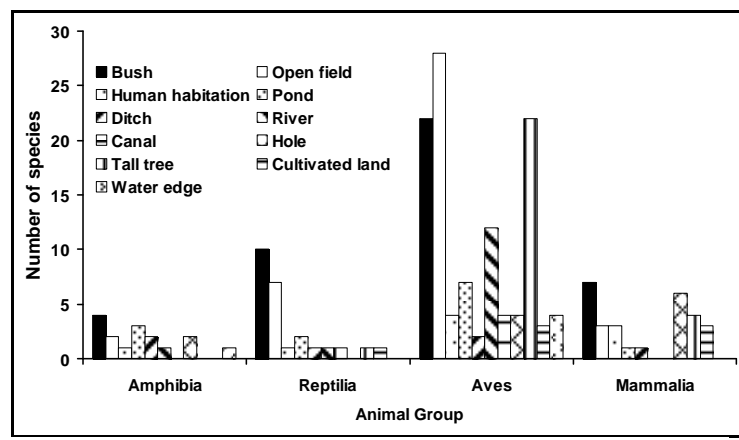

Fig. 5. Microhabitat utilization by wildlife species (Amphibian - Mammalian) in Eco park.

Table 1. Species composition, relative abundance, IUCN Red Book category population density and national status of wildlife in the Eco-Park.

\begin{tabular}{|c|c|c|c|c|c|c|}
\hline Scientific Name & English Name & Local Name & RA & PD/ha & $\begin{array}{c}\text { IUCN Local } \\
\text { status }\end{array}$ & HU \\
\hline \multicolumn{7}{|l|}{ Class: Amphibia } \\
\hline $\begin{array}{l}\text { Hoplobatrachus } \\
\text { tigerinus }\end{array}$ & Bull Frog & $\begin{array}{l}\text { Shonabang/ } \\
\text { Kolabang }\end{array}$ & $\mathrm{VC}$ & 5.16 & LR & $\mathrm{OP}, \mathrm{P}, \mathrm{Dt}$ \\
\hline $\begin{array}{l}\text { Euplyctis } \\
\text { cyanophlyctis }\end{array}$ & Skipper Frog & Kotkoti Bang & $\mathrm{C}$ & 4.00 & LR & $\mathrm{R}, \mathrm{P}, \mathrm{We}$ \\
\hline Rana alticola & Boulenger's Frog & Pana Bang & $\mathrm{F}$ & 8.50 & VU & $\mathrm{Bh}, \mathrm{P}$ \\
\hline Bufo melanostictus & Common Toad & Kuna Bang & $\mathrm{VC}$ & 17.33 & LR & $\begin{array}{c}\mathrm{Bh}, \mathrm{Op}, \mathrm{Hh}, \mathrm{H} \text {, } \\
\mathrm{Dt}\end{array}$ \\
\hline Microhyla ornata & Ornate Microhylid & China Bang & $\mathrm{F}$ & 8.50 & VU & $\mathrm{Bh}, \mathrm{H}$ \\
\hline M. rubra & Red Microhylid & Red china Bang & $\mathrm{F}$ & 6.33 & VU & $\mathrm{Bh}$ \\
\hline \multicolumn{7}{|l|}{ Class: Reptilia } \\
\hline $\begin{array}{l}\text { Hemidactylus } \\
\text { flaviviridis }\end{array}$ & House Gecko & Tiktiki & $\mathrm{C}$ & 10.33 & LR & $\mathrm{Bh}, \mathrm{Op}, \mathrm{Hh}, \mathrm{Tt}$ \\
\hline H. brookii & Spotted House Lizard & Tiktiki & $\mathrm{C}$ & 20.50 & LR & $\mathrm{Bh}, \mathrm{Op}$ \\
\hline H. bowringii & Bowring's House Lizard & Tiktiki & $\mathrm{C}$ & 8.67 & VU & $\mathrm{Bh}$ \\
\hline Gekko gecko & Wall Gecko & Takkhak & $\mathrm{C}$ & 10.00 & VU & $\mathrm{Bh}, \mathrm{Op}$ \\
\hline
\end{tabular}

ECOPRINT VOL 20, 2013 


\begin{tabular}{|c|c|c|c|c|c|c|}
\hline Calotis versicolor & Common Garden Lizard & Rokto-chosa & $\mathrm{VC}$ & 9.67 & LR & $\mathrm{Bh}, \mathrm{Op}$ \\
\hline Mabuya carinata & Common Skink & Anjoni & $\mathrm{VC}$ & 8.67 & LR & $\mathrm{Bh}, \mathrm{Op}$ \\
\hline M. dissimilis & Stripped Skink & Anjoni & $\mathrm{C}$ & 5.33 & VU & $\mathrm{Bh}, \mathrm{Op}$ \\
\hline Varanus bengalensis & Bengal Monitor & Gui Shap & $\mathrm{FC}$ & 2.33 & VU & $\mathrm{Bh}, \mathrm{P}$ \\
\hline$V$. flavescens & Yellow Monitor & Holdy-gui & $\mathrm{F}$ & 1.00 & $\mathrm{EN}$ & $\mathrm{Bh}, \mathrm{Cl}$ \\
\hline Xenochorphis piscator & $\begin{array}{l}\text { Cheekered Keel back } \\
\text { snake }\end{array}$ & Dhora-shap & $\mathrm{C}$ & 2.67 & LR & $\mathrm{P}, \mathrm{C}, \mathrm{Dt}$ \\
\hline Amphiesma stolatum & $\begin{array}{l}\text { Stripped Keel } \\
\text { back Snake }\end{array}$ & Dhora-shap & $\mathrm{C}$ & 1.50 & LR & $\mathrm{Bh}, \mathrm{Op}$ \\
\hline \multicolumn{7}{|l|}{ Class: Aves } \\
\hline Ardeola grayii & Pond Heron & Kana Bok & $\mathrm{C}$ & 8.00 & LR & $\begin{array}{c}\text { Op, Cl, R, P, } \\
\text { Dt, We }\end{array}$ \\
\hline Bubulcus ibis & Cattle Egret & Go Bok & $\mathrm{FC}$ & 4.50 & LR & $\mathrm{R}, \mathrm{P}, \mathrm{C}$ \\
\hline Egretta intermedia & Intermediate Egret & Maijala Bok & $\mathrm{FC}$ & 6.83 & VU & Op, R, P \\
\hline E. garzetta & Little Egret & Jotti Bok & $\mathrm{C}$ & 16.33 & LR & $\mathrm{R}, \mathrm{C}$ \\
\hline Nycticorax nycticorax & Night Heron & $\begin{array}{l}\text { Nishi Bok / } \\
\text { Ratchora }\end{array}$ & $\mathrm{F}$ & 9.17 & EN & $\begin{array}{l}\mathrm{P}, \mathrm{C} \\
\mathrm{We}\end{array}$ \\
\hline Milvus migrans & Pariah Kite & Bhuban-cheel & $\mathrm{VC}$ & 22.50 & VU & $\mathrm{Op}, \mathrm{Hh}, \mathrm{Tt}$ \\
\hline M. lineatus & Large Pariah Kite & Cheel & $\mathrm{FC}$ & 2.00 & LR & Op \\
\hline Gyps bengalensis & $\begin{array}{l}\text { White-backed } \\
\text { Vulture }\end{array}$ & Shakun & $\mathrm{FC}$ & 3.83 & VU & $\mathrm{Op}, \mathrm{Cl}, \mathrm{Tt}$ \\
\hline Haliastur indus & Brahminy Kite & Lal-cheel & $\mathrm{C}$ & 2.16 & $\mathrm{DD}$ & $\mathrm{Op}, \mathrm{Cl}$ \\
\hline Gallicrex cinerea & Water-cock & Kora & $\mathrm{FC}$ & 3.00 & $\mathrm{CR}$ & $\mathrm{R}$ \\
\hline $\begin{array}{l}\text { Amaurornis } \\
\text { phoenicurus }\end{array}$ & $\begin{array}{l}\text { White-breasted } \\
\text { Water Hen }\end{array}$ & Dahuk & $\mathrm{C}$ & 3.00 & VU & $\mathrm{R}$ \\
\hline Sterna aurantia & River Tern & Gang-cheet & $\mathrm{FC}$ & 1.83 & LR & $\mathrm{R}$ \\
\hline S. albifrons & Little Tern & Khudey-gangcheel & $\mathrm{F}$ & 3.33 & LR & $\mathrm{R}$ \\
\hline Streptopelia decaocto & Ring Dove & Raj-ghugu & $\mathrm{C}$ & 17.17 & $\mathrm{EN}$ & $\mathrm{Bh}, \mathrm{Tt}$ \\
\hline S. tranquebarica & Red Turtle Dove & Lal-ghugu & $\mathrm{FC}$ & 13.50 & EN & $\mathrm{Tt}$ \\
\hline S. chinensis & Spotted Dove & Tila-ghugu & $\mathrm{VC}$ & 13.50 & & $\mathrm{Op}, \mathrm{Tt}$ \\
\hline Columba livia & Rock Pigeon & Jalali Kabutar & $\mathrm{C}$ & 33.50 & $\begin{array}{l}\text { LR } \\
\text { LR }\end{array}$ & $\underset{\mathrm{Tt}}{\mathrm{Bh}, \mathrm{Op}, \mathrm{Hh}, \mathrm{Cl} \text {, }}$ \\
\hline Psittacula krameri & $\begin{array}{l}\text { Rose-ringed } \\
\text { Parakeet }\end{array}$ & Tia & $\mathrm{VC}$ & 6.00 & EN & Op \\
\hline Clamator jacobinus & $\begin{array}{l}\text { Pied Crested } \\
\text { Cuckoo }\end{array}$ & Jhuti Kokil & $\mathrm{C}$ & 12.50 & EN & Op \\
\hline Cuculus varius & Common Hawk-cuckoo & Choggelo & $\mathrm{C}$ & 9.33 & LR & $\mathrm{Bh}, \mathrm{Tt}$ \\
\hline $\begin{array}{l}\text { Eudynamys } \\
\text { scolopaceus }\end{array}$ & Koel & Kokil & $\mathrm{C}$ & 9.83 & LR & $\mathrm{Tt}$ \\
\hline Centropus sinensis & Crow-pheasant & Kanakuwa & $\mathrm{FC}$ & 2.50 & LR & Op \\
\hline
\end{tabular}




\begin{tabular}{|c|c|c|c|c|c|c|}
\hline Tyto alba & Barn Owl & Laxmi-pencha & $\mathrm{FC}$ & 5.33 & $\mathrm{EN}$ & Op \\
\hline Athene brama & Spotted Owlet & Phuti-pencha & $\mathrm{C}$ & 7.83 & LR & $\mathrm{Op}, \mathrm{Tt}$ \\
\hline Apus affinis & House Swift & Ghorani-nakkati & $\mathrm{C}$ & 69.67 & DD & $\mathrm{Bh}, \mathrm{Op}, \mathrm{Tt}$ \\
\hline Ceryle rudis & $\begin{array}{l}\text { Lesser Pied } \\
\text { Kingfisher }\end{array}$ & $\begin{array}{l}\text { Pakra Mach } \\
\text { rangha }\end{array}$ & $\mathrm{FC}$ & 8.33 & LR & $\mathrm{R}, \mathrm{Dt}$ \\
\hline Alcedo atthis & Common Kingfisher & Choto Machranga & $\mathrm{FC}$ & 17.33 & LR & $\mathrm{H}, \mathrm{R}, \mathrm{P}$ \\
\hline A. meninting & $\begin{array}{l}\text { Blue-eard } \\
\text { Kingfisher }\end{array}$ & Mach-rangha & $\mathrm{FC}$ & 4.00 & LR & $\mathrm{P}, \mathrm{C}$ \\
\hline Halcyon smyrnensis & $\begin{array}{l}\text { White-breasted } \\
\text { Kingfisher }\end{array}$ & $\begin{array}{l}\text { Shet buk } \\
\text { Machrangha }\end{array}$ & $\mathrm{C}$ & 11.50 & LR & $\mathrm{R}, \mathrm{P}$ \\
\hline Merops philippnus & Blue-tailed Bee -eater & Nilez suichora & $\mathrm{FC}$ & 5.16 & $\mathrm{DD}$ & $\begin{array}{l}\mathrm{Bh}, \mathrm{Op}, \\
\mathrm{Cl}\end{array}$ \\
\hline M. orientalis & Green Bee-eater & Suichora & $\mathrm{C}$ & 30.00 & $\mathrm{DD}$ & $\mathrm{Bh}, \mathrm{Cl}$, \\
\hline Upupa epops & Hoopoe & Hudhud-pakhi & $\mathrm{C}$ & 9.33 & DD & Op \\
\hline $\begin{array}{l}\text { Megalaima } \\
\text { haemacephala }\end{array}$ & $\begin{array}{l}\text { Crimson breasted } \\
\text { Barbet }\end{array}$ & $\begin{array}{l}\text { Choto basanta } \\
\text { Bauri }\end{array}$ & $\mathrm{C}$ & 12 & LR & $\mathrm{Bh}, \mathrm{Op}$ \\
\hline M. asiatica & Blue-throated Barbet & $\begin{array}{l}\text { Nilgri-basanta } \\
\text { Bauri }\end{array}$ & $\mathrm{FC}$ & 11.50 & LR & Op \\
\hline Dendrocopos macei & $\begin{array}{l}\text { Fulvous breasted } \\
\text { pied wood pecker }\end{array}$ & $\begin{array}{l}\text { Badami buk } \\
\text { kaththokra }\end{array}$ & $\mathrm{C}$ & 10.33 & LR & $\mathrm{Tt}$ \\
\hline Dinopium benghalense & $\begin{array}{l}\text { Lesser-golden back wood } \\
\text { pecker }\end{array}$ & $\begin{array}{l}\text { Sonali pit } \\
\text { kaththokra }\end{array}$ & $\mathrm{FC}$ & 13.00 & LR & $\mathrm{Bh}, \mathrm{Tt}, \mathrm{H}$ \\
\hline Lanius schach & $\begin{array}{l}\text { Black-headed } \\
\text { Shrike }\end{array}$ & Kalashir-koshi & $\mathrm{C}$ & 15.33 & LR & $\mathrm{Bh}$ \\
\hline Hirundo rustica & $\begin{array}{l}\text { Common } \\
\text { Swallow }\end{array}$ & Ababil Pakhi & $\mathrm{C}$ & 7.17 & LR & $\mathrm{We}$ \\
\hline Oriolus oriolus & Golden Oriole & $\begin{array}{l}\text { Sonali-halud } \\
\text { Pakhi }\end{array}$ & $\mathrm{C}$ & 8.67 & $\mathrm{DD}$ & $\mathrm{Bh}, \mathrm{Tt}$ \\
\hline O. xanthornus & $\begin{array}{l}\text { Black headed } \\
\text { Oriole }\end{array}$ & Holdey pakhi & $\mathrm{C}$ & 15.00 & LR & $\mathrm{Tt}$ \\
\hline Dicrurus macrocerus & Black Drongo & Fingey & $\mathrm{VC}$ & 59.67 & $\mathrm{DD}$ & $\mathrm{Op}, \mathrm{Cl}$ \\
\hline D. leucophaeus & Ashy Drongo & $\begin{array}{l}\text { Dhusar- } \\
\text { fingey }\end{array}$ & $\begin{array}{l}\mathrm{F} \\
\mathrm{C}\end{array}$ & 2.83 & LR & $\mathrm{Op}, \mathrm{Tt}$ \\
\hline D. aeneus & Bronzed Drongo & Choto-fingey & $\mathrm{C}$ & 8.50 & LR & $\mathrm{Bh}, \mathrm{Op}$ \\
\hline Sturnus malabaricus & Grey-headed Myna & Kath-shalik & $\mathrm{C}$ & 15.83 & LR & $\begin{array}{c}\mathrm{Bh}, \mathrm{Op}, \mathrm{Hh}, \mathrm{Cl} \text {, } \\
\text { Tt, H }\end{array}$ \\
\hline S. contra & Pied Myna & Gobrey-shalik & $\mathrm{VC}$ & 68.83 & LR & $\begin{array}{c}\mathrm{Bh}, \mathrm{Op}, \mathrm{Hh}, \mathrm{Cl} \text {, } \\
\mathrm{Tt}, \mathrm{H}\end{array}$ \\
\hline Acridotheres tristis & Common Myna & Bhat-shalik & $\mathrm{VC}$ & $\begin{array}{c}110.8 \\
3\end{array}$ & LR & $\mathrm{Bh}, \mathrm{Op}, \mathrm{Cl}$ \\
\hline A. ginginianus & Bank Myna & Gang-shalik & $\mathrm{C}$ & 18.67 & DD & $\mathrm{R}, \mathrm{We}$ \\
\hline A. fuscus & Jungle Myna & Jhuti-shalik & $\mathrm{C}$ & 57.17 & LR & $\mathrm{Bh}, \mathrm{Tt}$ \\
\hline
\end{tabular}

ECOPRINT VOL 20, 2013 


\begin{tabular}{|c|c|c|c|c|c|c|}
\hline $\begin{array}{l}\text { Dendrocitta } \\
\text { vagabunda }\end{array}$ & Rufous Treepie & Harichacha & $\mathrm{VC}$ & 8.67 & LR & $\mathrm{Bh}, \mathrm{Tt}$ \\
\hline Corvus splendens & House Crow & Kak-kowa & $\mathrm{VC}$ & 71.00 & LR & $\mathrm{Bh}, \mathrm{Tt}$ \\
\hline C. macrorhynchos & Jungle Crow & Dar-kak & $\mathrm{VC}$ & 18.00 & LR & $\mathrm{Bh}, \mathrm{Op}, \mathrm{Tt}$ \\
\hline \multirow[t]{2}{*}{ Pycnonotus cafer } & Red-vented & Kala-bulbul & $\mathrm{VC}$ & 86.50 & LR & $\mathrm{Bh}, \mathrm{Op}$ \\
\hline & Bulbul & & & & & \\
\hline Copsychus saularis & Magpie-robin & Doyel & $\mathrm{VC}$ & 97.5 & LR & $\mathrm{Bh}$ \\
\hline Passer domesticus & House Sparrow & Churoi & $\mathrm{VC}$ & $\begin{array}{c}125.1 \\
7\end{array}$ & LR & $\mathrm{Bh}, \mathrm{Op}, \mathrm{Cl}$ \\
\hline P. montanus & Tree Sparrow & Gecho-churoi & $\mathrm{VC}$ & 9.67 & LR & $\mathrm{Bh}, \mathrm{Op}, \mathrm{Tt}$ \\
\hline Ploceus philippinus & Baya & Babui & $\mathrm{VC}$ & 28.00 & LR & $\mathrm{Cl}$ \\
\hline \multicolumn{7}{|l|}{ Class: Mammalia } \\
\hline Suncus murinus & Whit- tailed Shrew & Chika & $\mathrm{C}$ & 8.33 & LR & $\mathrm{Op}, \mathrm{Tt}, \mathrm{H}$ \\
\hline Pteropus giganteus & Flying-fox & Badur & $\mathrm{C}$ & 9.33 & LR & $\mathrm{Tt}$ \\
\hline $\begin{array}{l}\text { Pipistrellus } \\
\text { coromandra }\end{array}$ & Pipistrelle & Chamchika & $\mathrm{FC}$ & 5.17 & LR & $\mathrm{B}, \mathrm{Tt}$ \\
\hline Tylonycteris pachypus & Club-footed Bat & Chamchika & $\mathrm{FC}$ & 14.16 & LR & $\mathrm{Bh}$ \\
\hline Canis aureus & Asiatic Jackal & Shial & $\mathrm{FC}$ & 1.33 & VU & $\mathrm{Bh}, \mathrm{Op}, \mathrm{H}$ \\
\hline Vulpes bengalensis & Bengal Fox & Khek-shial & $\mathrm{FC}$ & 1.33 & EN & $\mathrm{H}$ \\
\hline $\begin{array}{l}\text { Herpestes } \\
\text { auropunctatus }\end{array}$ & Small Mongoose & Choto-beji & $\mathrm{F}$ & 0.83 & $\mathrm{EN}$ & $\mathrm{Tt}$ \\
\hline Felis chaus & Jungle Cat & Ban-biral & $\mathrm{FC}$ & 1.33 & VU & $\mathrm{Bh}, \mathrm{Dt}$ \\
\hline Lepus nigricollis & Black-naped Here & Khargosh & $\mathrm{O}$ & 0.83 & VU & $\mathrm{H}$ \\
\hline Rattus rattus & Black Rat & Indur & $\mathrm{C}$ & 19.00 & LR & $\mathrm{Bh}, \mathrm{Cl}$ \\
\hline \multirow[t]{2}{*}{ R. norvegicus } & Brown Rat & Dhamshi & $\mathrm{FC}$ & 10.50 & DD & $\mathrm{Bh}, \mathrm{Cl}$ \\
\hline & & Indur & & & & \\
\hline R. fulvescens & Short Tail Rat & Ghashua Indur & $\mathrm{FC}$ & 4.33 & DD & $\mathrm{Cl}$ \\
\hline Mus musculus & House Rat & Nengti indur & $\mathrm{FC}$ & 2.50 & LR & $\mathrm{Hh}, \mathrm{H}$ \\
\hline M. booduga & Little Field Mouse & Metho-indur & $\mathrm{FC}$ & 8.83 & LR & $\mathrm{Hh}, \mathrm{H}, \mathrm{p}$ \\
\hline Bandicota indica & Larges Bandicoot Rat & Indur & $\mathrm{FC}$ & 5.83 & LR & $\mathrm{Op}, \mathrm{Hh}$ \\
\hline $\begin{array}{l}\text { Calloscirus } \\
\text { pygerythrus }\end{array}$ & Irrawaddy Squirrel & Badami-katbirali & $\mathrm{FC}$ & 2.33 & VU & $\mathrm{Bh}$ \\
\hline
\end{tabular}

Abbreviation: RA = Relative Abundance, $\mathrm{PD}=$ Population Density, HU = Habitat Utilization, VC = Very Common, $\mathrm{C}=$ Common, $\mathrm{F}=\mathrm{Few}, \mathrm{O}=$ Occasional, $\mathrm{CR}=$ Critically Endangered, $\mathrm{EN}=$ Endangered, $\mathrm{VU}=\mathrm{Vulnerable}, \mathrm{LR}=$ Lower Risk, $\mathrm{DD}=$ Data Deficient, $\mathrm{M}=$ Migratory, $\mathrm{R}=$ Resident, $\mathrm{Bh}=\mathrm{Bush}, \mathrm{Op}=$ Open place, $\mathrm{Hh}=\mathrm{Human}$ habitation, $\mathrm{Cl}=$ Cultivated land, $\mathrm{Tt}=$ Tall tree, $\mathrm{H}=$ Hole, $\mathrm{R}=$ River, $\mathrm{P}=\mathrm{Pond}, \mathrm{C}=\mathrm{Canal}, \mathrm{Dt}=\mathrm{Ditch}, \mathrm{We}=\mathrm{Water}$ edge.

\section{CONSERVATION ISSUES}

Every year, cyclones destroy the nesting and feeding grounds of wild animals, particularly for birds in the area. The requirement of fire wood and timber for the large number of inhabitants in the study area is a big threat for reducing the park areas. Most of the local people, particularly the poor people depend on the forest resources. As a result, nesting trees are being destroyed day by day. The population of dolphins, migratory birds and other aquatic birds in the Jamuna has been reduced enormously due to oil pollution, movement of people and repairing of fishing nets by the fishermen along the shore that create problem for the movement of birds. Grazing of domestic animals and exploitation of natural 
resources along the river shore might have created problem for feeding and roosting of birds and other wildlife.

\section{RECOMMENDATIONS}

Regular patrolling by trained persons should control unwise and illegal exploitation of forest resources. Plantation of indigenous fruit-trees with the sharing involvement of local people may increase food availability for wild animal, particularly for frugivores. Trapping and shooting must be prohibited. Oil pollution and movement of fishing boats should be reduced. Use of highly poisonous insecticides in the agricultural fields adjacent to the study area should not be allowed. Establishment of wildlife training centers is necessary where local people would be trained up and can play an effective role to aware people about the value of wild animals and their importance. Wildlife Protection Law 1974 should be strictly implemented through the local enforcement agencies. Shore and new char land areas 'Musa' must be protected for the migratory birds, fishes and other animals.

\section{ACKNOWLEDGEMENT}

The authors are grateful to the authority of Jamuna Eco-park, Sirajgonj for their permission and providing facilities during study period.

\section{REFERENCES}

Akter, S. 1977. The Wildlife Fauna of Baldah Garden, Dhaka (with notes on ecology habitat and present status) M.Sc. thesis (unpublished), Department of Zoology, University of Dhaka, Bangladesh.

Ali, S. and S.D. Ripley. 1968-1974. The Books of the Bird of India and Pakistan. Vols. 1-10. Oxford University Press. Bombay, London, New York.
Ali, S. and S.D. Ripley. 1983. A Pictorial Guide to the Birds of the Indian Subcontinent. Bombay Nat. Hist. Soc., Bombay, India.

Banerjee, R.K. 1978. Status and Composition of Avifauna of the Curzon Hall Campus and Ramna Park, Dhaka. M.Sc. thesis (unpublished), Department of Zoology, University of Dhaka, Bangladesh.

Daniel, J.C. 1983. The Book of Indian Reptiles. Bombay Nat. Hist. Soc., India, pp. 143.

Dunbar, R. and P. Dunbar. 1975. Social dynamics of gelada baboons. Contrib Primatol. 6:1-157.

Haque, M. N. 1975. The Avifauna of Madhupur Forest (with notes on ecology, status, distribution and food) M.Sc. thesis (unpublished), Department of Zoology, University of Dhaka, Bangladesh.

Hossain, M.L. and S.U. Sarker. 1997. Birds of Hatiya Islan, Noakhali, Bangladesh. Dhaka Univ. J. Biol.. Sci. 6(1):39-48.

Husain, K.Z. and S.U. Sarker. 1971. Notes on a collection of birds from Pabna. J. Asiat. Soc. Bangladesh 16(2):259-289.

Husain. K.Z. and S.U. Sarker. 1979. Notes on A collection of birds from Pabna. J. Asiat. Soc. Bangladesh 5(1): 15-24.

IUCN. 2000. Red Data Book of Threatened Birds of Bangladesh. The World Conservation Union, Dhaka, Bangladesh.

Jaman, M.F., M.S.Z. Haque and S.U. Sarker. 2004. Ecology, conservation problems and status of avifauna of Noakhali Charbata coastal area. J. NOAMI 21(1):1-13.

Jasmin, H. 1996. The Wildlife Fauna of Bangladesh Rifles Head Quarter, Dhaka. M.Sc. thesis (unpublished), Department of Zoology, University of Dhaka, Bangladesh.

Penafiel, S.R. 1995. Wildlife of Sundarbans. Forest Resources Management Project, Mandala 
Agricultural Development Corporation (MADECOR), Dhaka, Bangladesh, pp. 28-41.

Rosario, E.A. and S.K. Hai. 1996. List of Amphibians, Reptiles, Birds and Mammals in the Sundarbans Natural Mangrove Stand and Adjacent Coastal Areas. Forest Resources Management Project, Mandala Agricultural Development Corporation (MADECOR), Dhaka, Bangladesh, pp. 126-135.

Sarker, S.U. and N.J. Sarker. 1983. Endangered wildlife of Bangladesh. Tigerpaper 10(2):2628.

Sarker, S.U. and N.J. Sarker. 1985. Reptiles of Bangladesh. Tigerpaper 12(2):6-12.
Sarker, S.U. and N.J. Sarker. 1988. Wildlife of Bangladesh (A systematic list with the status and distribution). The Rico Printers, Nilkhet, Dhaka, $59 \mathrm{pp}$.

Sarker, S.U., M.F. Jaman, M.L. Hossain and N.J. Sarker. 2001. Wildlife diversity of Maheskhali Island: their ecology and conservation issues. J. NOAMI 18(1):17-31.

Smith, M.A. 1931. The Fauna of British India including Ceylon and Burma (Reptilia and Amphibia) Vol. 1, Taylor and Francis, London, 185 pp.

Smith, M.A. 1943. The Fauna of British India (Reptiles and Amphibian) Vol. 3, Serpents, Taylor and Francis, London. 